\title{
Hodges-Lehmann optimality of tests
}

\author{
Wilbert C.M. Kallenberg \\ Department of Applied Mathematics, University of Twente, Enschede, Netherlands
}

\author{
Stavros Kourouklis \\ Department of Mathematics, Faculty of Sciences, University of Patras, Patras, Greece
}

Received February 1991

Revised September 1991

\begin{abstract}
At several places in the literature there are indications that many tests are optimal in the sense of Hodges-Lehmann efficiency. It is argued here that shrinkage of the acceptance regions of the tests to the null set in a coarse way is already enough to ensure optimality. This type of argument can be used to show optimality of e.g. Kolmogorov-Smirnov tests, Cramér-von Mises tests, and likelihood ratio tests and many other tests in exponential families.
\end{abstract}

AMS 1980 Subject Classifications: Primary 62G20; Secondary 62F05, 60F10.

Keywords: Hodges-Lehmann efficiency, large deviations, Kullback-Leibler information number, exponential family, goodness-of-fit tests.

\section{Introduction}

There are many ways to describe the relative performance of test procedures in the case of large sample size. An overview of six of such methods is given in Serfling (1980, Chapter 10). One of these was introduced by Hodges and Lehmann (1956). Keeping the alternative fixed, two competing tests are compared with respect to the rate at which the probability of the error of the second kind tends to 0 , while both tests have the same fixed size. In a sense this is dual to the more familiar notion of Bahadur efficiency. Although the latter was introduced as 'stochastic comparison' based on the behaviour of the level attained or $p$-value, when considering convergence in distribution of attained levels, Bahadur efficiency may be described in the same way as Hodges-Lehmann efficiency above, changing the roles of the probability of the error of the second kind and the size (cf., e.g., Chandra and Ghosh (1978), Bahadur and Gupta (1986), Kallenberg (1981, 1983)).

Optimality in the sense of Bahadur efficiency is rather scarce. It seems to be restricted to tests which are not too much different from either likelihood ratio tests or tests based on Kullback-Leibler information numbers. On the other hand there are several places in literature with indications that many tests are optimal in the sense of Hodges-Lehmann efficiency. In Tusnády (1977, p.391) it is stated in a particular example that "the majority of tests of rate $A=0$ for this problem are ERO (exponential rate optimal) at any $Q$ " (ERO with $A=0$ corresponds to optimal in the sense of Hodges-Lehmann efficiency). Nikitin (1987, p.78) remarks that a lot of the tests he considers are Hodges-Lehmann optimal 
in contrast to the Bahadur case. Raghavachari (1983) presents an example where the ranks are asymptotically fully informative in Hodges-Lehmann efficiency sense but not in the Bahadur efficiency sense. Further a number of other examples can be found in Baringhaus (1987) and Kourouklis (1988).

It is the purpose of this note to show that the optimality is due to shrinking of the acceptance regions of the tests to the null set in a coarse way. This basic convergence is cnough to cnsure Hodges-Lehmann optimality. The same argument is used to show that in regular exponential families many tests including the likelihood ratio test are optimal in the sense of Hodges-Lehmann efficiency. In fact it is enough that the limit of the acceptance regions is not closer to the alternative than the null set in terms of Kullback-Leibler information numbers. On the other hand if a substantial part of the acceptance region comes closer to the alternative than the null set, then Hodges-Lehmann optimality tails.

\section{Basic result}

Let $\mathscr{S}$ be a Hausdorff space and let $\mathscr{B}$ be the $\sigma$-field of Borel sets in $\mathscr{S}$. Let $A$ be the set of all probability measures on $\mathscr{B}$. For $P, Q \in \Lambda$ the Kullback-Leibler information number $K(Q, P)$ is defined by

$$
K(Q, P)= \begin{cases}E_{Q} \log \frac{\mathrm{d} Q}{\mathrm{~d} P} & \text { if } Q \ll P \\ \infty & \text { otherwise }\end{cases}
$$

For any subset $\Omega$ of $A$ and any $P \in A$ define

$$
K(\Omega, P)=\inf \{K(Q, P): Q \in \Omega\}
$$

with, by convention, $K(\Omega, P)=\infty$ if $\Omega$ is empty.

Let $X_{1}, X_{2}, \ldots$ be a sequence of i.i.d. r.v.'s taking values in $\mathscr{S}$ according to $P \in \Lambda$. For each positive integer $n$ the empirical probability measure based on $X_{1}, \ldots, X_{n}$ is denoted by $\hat{P}_{n}$. Consider the testing problem $\mathrm{H}_{0}: P \in \Lambda_{0}$ against the alternative $\mathrm{H}_{1}: P \in \Lambda_{1}$, where $\Lambda_{0}, \Lambda_{1} \subset \Lambda, \Lambda_{0} \cap \Lambda_{1}=\emptyset$. Let $\phi_{n}=$ $\psi_{n}\left(x_{1}, \ldots, x_{n}\right)$ denote a (randomized) test function and let

$$
\alpha_{n}=\sup \left\{E_{P} \phi_{n}: P \in \Lambda_{0}\right\}, \quad \beta_{n}(P)=E_{P}\left(1-\phi_{n}\right) .
$$

We consider tests of asymptotic level- $\alpha$, i.e.

$$
\lim _{n \rightarrow \infty} \alpha_{n}=\alpha,
$$

where $0<\alpha<1$ is kept fixed. If for $P \in \Lambda_{1}$ there exists $d=d(P) \in(0, \infty)$ such that

$$
\lim _{n \rightarrow \infty} n^{-1} \log \beta_{n}(P)=-\frac{1}{2} d(P),
$$

then $d(P)$ is called the Hodges-Lehmann index of $\left\{\phi_{n}\right\}$ at $P$. By the well-known Stein's lemma, cf. Bahadur (1971) or Kourouklis (1988), we have

$$
\liminf _{n \rightarrow \infty} n^{-1} \log \beta_{n}(P) \geqslant-K\left(\Lambda_{0}, P\right)
$$

In view of (6) a sequence of tests $\left\{\phi_{n}\right\}$ is called Hodges-Lehmann asymptotically optimal at the alternative $P$ if its Hodges-Lehmann index equals $2 K\left(\Lambda_{0}, P\right)$. 
Consider now tests $\phi_{n}$ based on test statistics $T\left(\hat{P}_{n}\right)$, rejecting $\mathrm{H}_{0}$ for large values of the test statistics, i.e.

$$
\phi_{n}\left(X_{1}, \ldots, X_{n}\right)= \begin{cases}1 & \text { if } T\left(\hat{P}_{n}\right)>c_{n} \\ \gamma_{n} & \text { if } T\left(\hat{P}_{n}\right)=c_{n}, \\ 0 & \text { if } T\left(\hat{P}_{n}\right)<c_{n}\end{cases}
$$

where $T$ is an extended real-valued function on $\Lambda$. Denoting the topology of convergence on all Borel sets by $\tau$, it is assumed that $T$ is $\tau$-continuous at each $Q \in \Lambda$ with $K(Q, P)<\infty$ (cf. Groeneboom et al. (1979), p.554). Inspection of the proof of Theorem 3.2 in Groeneboom et al. (1979) yields for each $r \in \mathbb{R}$ and sequence of real numbers $\left\{u_{n}\right\}$ with $\lim _{n \rightarrow \infty} u_{n}=0$,

$$
\begin{aligned}
& \underset{n \rightarrow \infty}{\lim \sup } n^{-1} \log P\left[T\left(\hat{P}_{n}\right) \leqslant r+u_{n}\right] \\
& \quad=\lim \sup _{n \rightarrow \infty} n^{-1} \log P\left[-T\left(\hat{P}_{n}\right) \geqslant-r-u_{n}\right] \leqslant-K\left(\Omega_{r}, P\right),
\end{aligned}
$$

where $\Omega_{r}=\{Q \in A: T(Q) \leqslant r\}$.

In many cases $n^{1 / 2} T\left(\hat{P}_{n}\right)$ has a limiting distribution under $\mathrm{H}_{0}$ and therefore the critical value $c_{n}$ converges to 0 , implying by (8),

$$
\limsup _{n \rightarrow \infty} n^{-1} \log \beta_{n}(P) \leqslant \limsup _{n \rightarrow \infty} n^{-1} \log P\left[T\left(\hat{P}_{n}\right) \leqslant c_{n}\right] \leqslant-K\left(\Omega_{0}, P\right) .
$$

Moreover, often $\{Q \in \Lambda: T(Q) \leqslant 0\}=\Lambda_{0}$ and hence $K\left(\Omega_{0}, P\right) \geqslant K\left(\Lambda_{0}, P\right)$. Together with (6) and (9) this yields that the Hodges-I ehmann index equals $K\left(A_{0}, P\right)$ and hence the test is Hodges-Lehmann optimal.

Thus we have proven the following theorem.

Theorem 2.1. Let $\phi_{n}$ be given by (7) and satisfy (4). Let $T$ be $\tau$-continuous at each $Q \in \Lambda$ with $K(Q, P)<\infty$. Assume that $\lim _{n \rightarrow \infty} c_{n}=0$ and

$$
\{Q \in A: T(Q) \leqslant 0\}=\Lambda_{0},
$$

then $\left\{\phi_{n}\right\}$ is Hodges-Lehmann asymptotically optimal at the alternative $P$.

Application of this theorem leads to optimality of (weighted) Kolmogorov-Smirnov statistics, (generalized) Cramćr-von Mises statistics and the (generalized) Watson statistic in testing goodness-of-fit, cf. Nikitin (1987).

Remark 2.1. Generalization of Theorem 2.1 to the $k$-sample case can be done similarly as in Nikitin (1987).

Consistency of a test sequence $\left\{\phi_{n}\right\}$ of the form (7) at $Q$ typically corresponds to $T(Q)>0$. Condition (10) may fail, because $\left\{\phi_{n}\right\}$ is not consistent at certain alternatives. Sometimes it seems to be more natural to add these alternatives to the null hypothesis, cf. Remark 2.2. For tests which are consistent at each alternative, we thus usually have $T(Q)>0$ for all $Q \in \Lambda_{1}$. Somctimcs nevertheless (10) does not hold, due to the fact that $\Lambda_{1} \neq \Lambda-\Lambda_{0}$. We present two typical examples. 
Example 2.1. Let $\mathscr{S}=[0,1]$ and $\Lambda_{0}=\left\{Q_{0}\right\}$ with $Q_{0}$ the uniform distribution on $[0,1]$. Let $A_{1}, \ldots, A_{k}$ be a partition of $[0,1]$. The chi-square statistic is of the form (7) with

$$
T(Q)=\sum_{i=1}^{k} \frac{\left\{Q\left(A_{i}\right)-Q_{0}\left(A_{i}\right)\right\}^{2}}{Q_{0}\left(A_{i}\right)}
$$

It is obvious that the chi-square test is not optimal at $P$ for testing $Q=Q_{0}$ against $Q \neq Q_{0}$ for $P \neq Q_{0}$ with $P\left(A_{i}\right)=Q_{0}\left(A_{i}\right)$. However, taking

$$
\Lambda_{0}=\left\{Q \in \Lambda: Q\left(A_{i}\right)=Q_{0}\left(A_{i}\right), i=1, \ldots, k\right\},
$$

Theorem 2.1 ensures the optimality of the chi-square test. Also in the 'multinomial setting', i.e. replacing $\mathscr{S}=[0,1]$ by $\mathscr{S}=\{1, \ldots, k\}$, the chi-square test is optimal.

Remark 2.2. If the practical situation at hand justifies it, one would like to take $\{Q \in \Lambda: T(Q) \leqslant 0\}$ as the null hypothesis to test when using (7) with a critical value tending to 0 . For instance, in Example 2.1 one could think of the wider null hypothesis $\left\{Q \in \Lambda: Q\left(A_{i}\right)=Q_{0}\left(A_{i}\right), i=1, \ldots, k\right\}$ instead of $\left\{Q_{0}\right\}$.

Example 2.2. Let $\mathscr{S}=[0,1]$ and $\Lambda_{0}=\left\{Q_{0}\right\}$ with $Q_{0}$ the uniform distribution on $[0,1]$. Let $\Lambda_{1}=$ $\{Q \in A: T(Q)>0\}$, where $T(Q)=\sup _{x}\{Q([0, x])-x\}$, the one-sided Kolmogorov-Smirnov statistic. Taking $\phi_{n}$ of the form (7) with $T(Q)$ as above, it is well known that the one-sided Kolmogorov-Smirnov statistic is not for all $P \in \Lambda_{1}$ optimal although the test is consistent at any $P \in \Lambda_{1}$, cf. Nikitin (1987, p.84). This is due to the fact that there exist $P \in \Lambda_{1}$ and $Q \in \Lambda$ such that $T(Q)=0$ and $K(Q, P)<K\left(\Lambda_{0}, P\right)$.

Remark 2.3. It is easily seen that (10) may be replaced by the weaker condition $\Omega_{0} \subset \Lambda_{0}$, or even

$$
K\left(\Omega_{0}, P\right) \geqslant K\left(\Lambda_{0}, P\right) .
$$

However, in applications we usually meet (10) and therefore the theorem is presented in this form.

Remark 2.4. Condition (10) is the technical expression for what is called in the introduction "shrinking of the acceptance region to the null set in a coarse way". The word coarse is used, because not the limiting distribution of something like $\sqrt{n} T\left(\hat{P}_{n}\right)$ is involved, but only basic convergence.

Remark 2.5. Although the one-sided Kolmogorov-Smirnov test seems to be more appropriate for testing $\Lambda_{0}$ against $\Lambda_{1}$ in Example 2.2, the two-sided Kolmogorov-Smirnov test is optimal at any $P \in \Lambda_{1}$, and in general the one-sided Kolmogorov-Smirnov test is not. This phenomenon is similar to Brown's (1971) heuristic principle 1 (forget 'extra' information about the alternative hypothesis) and may be understood by realizing that enlarging the alternative hypothesis to $\Lambda-\Lambda_{0}$ usually implies $T(Q)>0$ at each $Q \in \Lambda-\Lambda_{0}$ for the new test (consistency), while the change in the acceptance region in the neighbourhood of the null set is of a local character, which is not picked up by the criterion of Hodges-Lehmann efficiency.

We close this section with another application of Theorem 2.1, showing the optimality of a test, which is close to Wilcoxon's one-sample test.

Example 2.3. Let $\mathscr{S}=\mathbb{R}$ and $\Lambda_{0}=\{Q \in \Lambda: T(Q) \leqslant 0\}$, where

$$
T(Q)=\iint\left\{h(x, y)-\frac{1}{2}\right\} \mathrm{d} Q(x) \mathrm{d} Q(y)
$$


with

$$
h(x, y)= \begin{cases}1 & \text { if } x+y>0 \\ 0 & \text { if } x+y \leqslant 0 .\end{cases}
$$

Consider the test of the form (7) with this $T$. Note that this test is closely related to Wilcoxon's one-sample test, which itself is not of the form (7), since $T$ in (7) may not depend on $n$, cf. e.g. Serfling (1980, p.174). Since $T$ is continuous at each $Q$ with respect to the topology induced by the supremum metric, $T$ is also $\tau$-continuous at each $Q$ (cf. Groeneboom et al. (1979), p.555). Further it is easily seen that $\lim _{n \rightarrow \infty} c_{n}=0$ and therefore, by direct application of Theorem 2.1, the Hodges-Lehmann asymptotical optimality of the test at each alternative $P$ is obtained.

\section{Exponential families}

Let $X_{1}, X_{2}, \ldots, X_{n}$ be i.i.d. r.v.'s each distributed according to an exponential family

$$
\mathrm{d} P_{\theta}(x)-\exp \left\{\theta^{\prime} x-\psi(\theta)\right\} \mathrm{d} \mu(x), \quad \theta \in \Theta \subset \mathbb{R}^{k}, x \in \mathbb{R}^{k},
$$

where $\mu$ is a $\sigma$-finite non-degenerate measure, $\Theta$ denotes the natural parameter space, i.e. $\Theta=$ $\left\{\theta \in \mathbb{R}^{k}: \int \exp \left(\theta^{\prime} x\right) \mathrm{d} \mu(x)<\infty\right\}$, and

$$
\psi(\theta)=\log \int \exp \left(\theta^{\prime} x\right) \mathrm{d} \mu(x), \quad \theta \subset \Theta .
$$

For $\theta \in \Theta^{*}=\left\{\theta \in \Theta: E_{\theta}\left\|X_{1}\right\|<\infty\right\}$ define

$$
\lambda(\theta)=E_{\theta} X_{1} .
$$

The mapping $\lambda$ is $1-1$ on $\Theta^{*}$ (cf. Lemma 2.2 in Berk (1972)) and $\lambda(\theta)=\operatorname{grad} \psi(\theta)$ if $\theta \in$ int $\Theta$. We write $K(\vartheta, \theta)$ for $K\left(P_{\vartheta}, P_{\theta}\right), K\left(\Theta_{0}, \theta\right)=\inf \left\{K(\vartheta, \theta): \vartheta \in \Theta_{0}\right\}$ and similarly $K\left(\vartheta, \Theta_{0}\right)$, where $\Theta_{0} \subset \Theta$. Further a sort of Kullback-Leibler 'distance' from the boundary of $\Theta$ to $\theta \in$ int $\Theta$ is defined by

$$
K(\theta)=\sup \{a \in \mathbb{R}:\{\vartheta \in \Theta: K(\vartheta, \theta) \leqslant a\} \text { is a compact subset of int } \Theta\} .
$$

Note that if $\Theta$ is open and $\left\{x \in \mathbb{R}^{\mathrm{k}}\right.$ : $\left.\sup \left\{\theta^{\prime} x-\psi(\theta): \theta \in \Theta\right\}<\infty\right\}$ is open, then $K(\theta)=\infty$, cf. Kourouklis (1984). Now we have the following result.

Theorem 3.1. Let $\left\{\phi_{n}\right\}$ be a sequence of tests based on $\bar{X}_{n}$ for testing $\mathrm{H}_{0}: \theta \in \Theta_{0}$ against $\mathrm{H}_{1}: \theta \in \Theta_{1} \subset$ $\Theta-\Theta_{0}$, satisfying (4). Let $\theta \in($ int $\Theta) \cap \Theta_{1}$. Suppose that $K\left(\Theta_{0}, \theta\right) \leqslant K(\theta)$.

Assume that for each $0<\varepsilon<K\left(\Theta_{0}, \theta\right)$ there exists $N_{\varepsilon}$ such that for all $n \geqslant N_{\varepsilon}$,

$$
\left\{x: \phi_{n}(x)=1\right\} \supset \lambda\left[\left\{\vartheta \in \Theta: K(\vartheta \theta)<K\left(\Theta_{0}, \theta\right)-\varepsilon\right\}\right] .
$$

Then $\left\{\phi_{n}\right\}$ is Hodges-Lehmann optimal at $\theta$.

Proof. Without loss of generality assume $K\left(\Theta_{0}, \theta\right)>0$. Let $0<\varepsilon<K\left(\Theta_{0}, \theta\right)$. By Lemma 3.2 in Kallenberg (1981) we obtain

$$
\begin{aligned}
\beta_{n}\left(P_{\theta}\right)=E_{0}\left(1-\phi_{n}\right) & \leqslant P_{\theta}\left[\phi_{n}\left(\bar{X}_{n}\right)<1\right] \\
& \leqslant P_{\theta}\left[\bar{X}_{n} \notin \lambda\left\{\vartheta \in \Theta: K(\vartheta, \theta)<K\left(\Theta_{0}, \theta\right)-\varepsilon\right\}\right] \\
& =\exp \left\{-n\left[K\left(\Theta_{0}, \theta\right)-\varepsilon\right]+O(\log n)\right\}
\end{aligned}
$$


as $n \rightarrow \infty$ and hence

$$
\limsup _{n \rightarrow \infty} n^{-1} \log \beta_{n}\left(P_{\theta}\right) \leqslant-K\left(\Theta_{0}, \theta\right)+\varepsilon .
$$

Since $0<\varepsilon<K\left(\Theta_{0}, \theta\right)$ was arbitrarily chosen, the Hodges-Lehmann optimality of $\left\{\phi_{n}\right\}$ at $\theta$ follows by (6) and (17).

Condition (15) holds if in $\lambda^{-1}$-space the acceptance region converges to $\Theta_{0}$ and this is satisfied for many tests, e.g. usually for the likelihood ratio test if $\Theta_{1}=\Theta-\Theta_{0}$. Define

$$
L(x)= \begin{cases}\sup _{\theta \in \Theta}\left\{\theta^{\prime} x-\psi(\theta)\right\}-\sup _{\vartheta \in \Theta_{0}}\left\{\vartheta^{\prime} x-\psi(\vartheta)\right\} & \text { if } \sup _{\vartheta \in \Theta_{0}}\left\{\vartheta^{\prime} x-\psi(\vartheta)\right\}<\infty \\ \infty & \text { otherwise }\end{cases}
$$

With this notation the size- $\alpha$ likelihood ratio test of $\mathrm{H}_{0}: \theta \in \Theta_{0}$ against $\mathrm{H}_{1}: \theta \in \Theta_{1}=\Theta-\Theta_{0}$ based on $n$ observations is given by

$$
\phi_{n}\left(\bar{x}_{n}\right)= \begin{cases}1 & \text { if } L\left(\bar{x}_{n}\right)>d_{n} \\ \delta_{n} & \text { if } L\left(\bar{x}_{n}\right)=d_{n} \\ 0 & \text { if } L\left(\bar{x}_{n}\right)<d_{n}\end{cases}
$$

where

$$
d_{n}=\inf \left\{d: \sup _{\vartheta \in \Theta_{0}} P_{\vartheta}\left[L\left(\bar{X}_{n}\right)>d\right] \leqslant \alpha\right\}
$$

and

$$
\delta_{n}=\sup \left\{\delta \in[0,1]: \sup _{\vartheta \in \Theta_{0}} E_{\vartheta} \phi_{n}\left(\bar{X}_{n}\right) \leqslant \alpha\right\}
$$

Corollary 3.2. Let $\left\{\phi_{n}\right\}$ be the likelihood ratio test for testing $\mathrm{H}_{0}: \theta \in \Theta_{0}$ against $\mathrm{H}_{1}: \theta \in \Theta_{1}=\Theta-\Theta_{0}$, satisfying (4). Suppose that the critical value $d_{n}\left(c f\right.$. (19)) satisfies $\lim _{n \rightarrow \infty} d_{n}=0$. Then the likelihood ratio test is Hodges-Lehmann optimal at each $\theta \in($ int $\Theta) \cap \Theta_{1}$ with $K\left(\Theta_{0}, \theta\right) \leqslant K(\theta)$.

Proof. If $\bar{x}_{n} \in \lambda\left(\Theta^{*}\right)$ then

$$
\phi_{n}\left(\bar{x}_{n}\right)= \begin{cases}1 & \text { if } K\left(\lambda^{-1}\left(\bar{x}_{n}\right), \Theta_{0}\right)>d_{n}, \\ \delta_{n} & \text { if } K\left(\lambda^{-1}\left(\bar{x}_{n}\right), \Theta_{0}\right)=d_{n}, \\ 0 & \text { if } K\left(\lambda^{-1}\left(\bar{x}_{n}\right), \Theta_{0}\right)<d_{n} .\end{cases}
$$

If $K\left(\Theta_{0}, \theta\right)=0$, Hodges-Lehmann optimality trivially holds. Therefore assume $K\left(\Theta_{0}, \theta\right)>0$. Let $0<\varepsilon<K\left(\Theta_{0}, \theta\right)$. The sets

$$
C=\left\{\vartheta \in \Theta: K(\vartheta, \theta) \leqslant K\left(\Theta_{0}, \theta\right)-\varepsilon\right\} \quad \text { and } \quad D=\left\{\vartheta \in \Theta: K(\vartheta, \theta)=K\left(\Theta_{0}, \theta\right)-\frac{1}{2} \varepsilon\right\}
$$

are compact subsets of int $\Theta$. For any $\vartheta \in C$ and $\theta_{0} \in \Theta_{0}$ there exists $t \in(0,1)$ such that $t \vartheta+(1-t) \theta_{0}$ $\in D$. Moreover,

$$
K\left(\vartheta, \theta_{0}\right) \geqslant K\left(\vartheta, t \vartheta+(1-t) \theta_{0}\right) \geqslant \inf \{K(\eta, D): \eta \in C\}>0 .
$$

Because $\vartheta \in C$ and $\theta_{0} \in \Theta_{0}$ are arbitrary chosen, wc havc inf $\left\{K\left(\vartheta, \Theta_{0}\right): \vartheta \in C\right\}>0$. Since $\lim _{n \rightarrow \infty} d_{n}=0$ condition (15) is fulfilled and Theorem 3.1 yields the result. 
Remark 3.1. The condition that the critical value of the likelihood ratio test tends to zero is usually fulfilled. For instance the following weak kind of similarity condition, which essentially states that the size (or a fixed part of it) is attained at parameter points bounded away from the boundary of the parameter space, is sufficient: there exists an $\varepsilon>0$ and a compact set $C \subset$ int $\Theta$ such that $\sup _{\theta \in \Theta_{0} \cap C} E_{\theta} \phi_{n} \geqslant \varepsilon \alpha_{n}$. Note that the case of a simple hypothesis is covered by it.

Remark 3.2. If $\Theta_{1} \subsetneq \Theta-\Theta_{0}$ optimality of the likelihood ratio test may fail. An example is given in Brown et al. (1984). However, if $\Theta_{1}$ is replaced by $\Theta-\Theta_{0}$ the likelihood ratio test is optimal in their example.

Remark 3.3. If the acceptance region shrinks to the null set in the sense that for any $\varepsilon>0$ and sufficiently large $n$,

$$
\left\{x: \phi_{n}(x)<1\right\} \subset\left\{x:\|x-\lambda(\vartheta)\| \leqslant \varepsilon, \vartheta \in \Theta_{0}\right\},
$$

then (15) holds.

Remark 3.4. If a substantial part of the acceptance region comes closer to the alternative than the null set, then Hodges-Lehmann optimality fails. More precisely, if for some $\varepsilon>0$ and all $n \geqslant N_{\varepsilon}$,

$$
\left\{x: \phi_{n}(x)=0\right\} \supset A
$$

for some open subset $A$ of $\lambda\left[\left\{\vartheta \in \Theta: K(\vartheta, \theta) \leqslant K\left(\Theta_{0}, \theta\right)\right\}\right]$, where $K\left(\Theta_{0}, \theta\right) \leqslant K(\theta)$, then

$$
\begin{aligned}
\liminf _{n \rightarrow \infty} n^{-1} \log \beta_{n}\left(P_{\theta}\right) & =\liminf _{n \rightarrow \infty} n^{-1} \log E_{\theta}\left(1-\phi_{n}\right) \\
& \geqslant \liminf _{n \rightarrow \infty} n^{-1} \log P_{\theta}\left(\bar{X}_{n} \in A\right) \geqslant-K\left(\lambda^{-1}(A), \theta\right)>-K\left(\Theta_{0}, \theta\right)
\end{aligned}
$$

and hence $\left\{\phi_{n}\right\}$ is not optimal.

We conclude with some examples.

Example 3.1 (comparison of Gauss test and t-test). Let $X_{1}, \ldots, X_{n}$ be i.i.d. r.v.'s with a normal $\mathrm{N}(0,1)$-distribution. Consider the testing problem $\mathrm{H}_{0}: \theta=0$ against $\mathrm{H}_{1}: \theta \neq 0$. Let $\left\{\phi_{n}^{(1)}\right\}$ be the Gauss test and $\left\{\phi_{n}^{(2)}\right\}$ the $t$-test. The Gauss test may be considered as the likelihood ratio test for the present testing problem, thus being optimal with Hodges-Lehmann index at $\theta$ equal to $d_{1}(\theta)=\frac{1}{2} \theta^{2}$. The $t$-test may also be considered as a likelihood ratio test, but for the testing problem $\mathrm{H}_{0}: \theta=0$ against $\mathrm{H}_{1}: \theta \neq 0$ when the observations are normally $\mathrm{N}\left(\theta, \sigma^{2}\right)$-distributed. Again we may apply Corollary 3.2 , leading to the Hodges-Lehmann index

$$
d_{2}(\theta)=\inf _{\sigma^{2}>0} E_{0, \sigma^{2}} \log \left(\mathrm{d} P_{0, \sigma^{2}} / \mathrm{d} P_{\theta, 1}\right),
$$

where $P_{\theta, \sigma^{2}}$ denotes the normal $\mathrm{N}\left(\theta, \sigma^{2}\right)$-distribution. It is easily seen that $d_{2}(\theta)=\frac{1}{2} \theta^{2}$, implying optimality of the $t$-test also in the present testing problem. So, contrary to comparison in the Bahadur case (cf. Bahadur (1971), p.30), there is no difference between the Gauss test and the $t$-test when using Hodges-Lehmann efficiency. The same phenomenon holds in the multivariate case, cf. Kourouklis (1988), p.92.

Example 3.2 (chi-square versus likelihood ratio). Let $X=\left(X_{1}, \ldots, X_{k}\right)$ have a multinomial distribution with parameters $n, p_{1}, \ldots, p_{k}\left(p_{i}>0, i=1, \ldots, k\right)$. Consider the testing problem $\mathrm{H}_{0}: p=p_{0}$ against $p \neq p_{0}$, where $p=\left(p_{1}, \ldots, p_{k}\right)$ and $p_{0}=\left(p_{01}, \ldots, p_{0 k}\right)$, the latter a given fixed point. The optimality of the likelihood ratio test follows e.g. from Corollary 3.2, while the optimality of the chi-square test was 
already established in Example 2.1. So again, both test are different in the Bahadur case (cf. Bahadur (1971), p.31) but are both optimal in the Hodges-Lehmann sense. In fact it is easily seen that any test of the form

$$
\phi_{n}(x)= \begin{cases}1 & \text { if } f\left(n^{-1} x, p_{0}\right)>d_{n} \\ \delta_{n} & \text { if } f\left(n^{-1} x, p_{0}\right)=d_{n} \\ 0 & \text { if } f\left(n^{-1} x, p_{0}\right)<d_{n}\end{cases}
$$

with $f\left(p, p_{0}\right)$ continuous in $p_{0}, f\left(p_{0}, p_{0}\right)=0<f\left(p, p_{0}\right)$ for all $p \neq p_{0}$, is Hodges-Lehmann optimal for testing $\mathrm{H}_{0}: p=p_{0}$ against $\mathrm{H}_{1}: p \neq p_{0}$. Note that the whole Cressie-Read class of tests (cf. Cressie and Read (1984)) is of the form (20).

The preceding example may be generalised in an obvious way to general exponential families and tests based on 'distances', showing that indeed many tests are optimal.

\section{References}

Bahadur, R.R. (1971), Some Limit Theorems in Statistics (SIAM, Philadelphia, PA).

Bahadur, R.R. and J.C. Gupta (1986), Distribution optimality and second-order efficiency of test procedures, in: J. van Ryzin, ed., Adaptive Statistical Procedures and Related Topics, IMS Lecture Notes 8, 315-331.

Baringhaus, L. (1987), Asymptotic optimality of multivariate linear hypothesis tests, J. Multivariate Anal. 23, 303-311.

Berk, R.H. (1972), Consistency and asymptotic normality of MLE's for exponential models, Ann. Math. Statist. 43, 193-204.

Brown, L.D. (1971), Non-local asymptotic optimality of appropriate likelihood ratio tests, Ann. Math. Statist. 42, 12061240.

Brown, L.D., F.H. Ruymgaart and D.R. Truax (1984), Hodges-Lehmann efficacies for likelihood ratio type tests in curved bivariate normal families, Statist. Neerlandica 38(2), 21-36.

Chandra, T.K. and J.K. Ghosh (1978), Comparison of tests with same Bahadur-efficiency, Sankhya Ser. A 40, 253-277.

Cressie, N. and T.R. Read (1984), Multinomial goodness-of-fit tests, J. Roy. Statist. Soc. Ser. B 46, 440-464.

Hodges, J.L. and E.L. Lehmann (1956), The efficiency of some nonparametric competitors of the $t$-test, Ann. Math. Statist. 27, 324-335.

Groeneboom, P., J. Oosterhoff and F.H. Ruymgaart (1979),

Large deviation theorems for empirical probability measures, Ann. Probab. 7, 553-586.

Kallenberg, W.C.M. (1981), Bahadur deficiency of likelihood ratio tests in exponential families, J. Multivariate Anal. 11, 506-531.

Kallenberg, W.C.M. (1983), Asymptotic efficiency and deficiency of tests, Proc. of the 44th. Session of the Internat. Statist. Inst. (Bulletin of the ISI) Vol. L., Bk. 2, pp. 11731189.

Kourouklis, S. (1984), A large deviation result for the likelihood ratio statistic in exponential families, Ann. Statist. 12, 1510-1521.

Kourouklis, S. (1988), Hodges-Lehmann efficacies of certain tests in multivariate analysis and regression analysis, Canad. J. Statist. 16, 87-95.

Nikitin, Y.Y. (1987), On the Hodges-Lehmann asymptotic efficiency of nonparametric tests of goodness-of-fit and homogeneity, Theory Probab. Appl. 32, 77-85.

Raghavachari, M. (1983), On the computation of HodgesLehmann efficiency of test statistics, in: P.J. Bickel, K. Doksum and J.L. Hodges, eds., A Festschrift for Erich $L$. Lehmann (Wadsworth, Belmont) pp. 367-378.

Serfling, R.J. (1980), Approximation Theorems of Mathematical Statistics (Wiley, New York).

Tusnády, G. (1977), On asymptotically optimal tests, Ann. Statist. 5, 385-393. 